\title{
Re-crystallisation of Amorphous Silicon in the Production of Low Defect Density Silicon on Sapphire Thin Films
}

\author{
W.R. McKenzie*, H. Domyo**, T. Ho** and P.R. Munroe* \\ * School of Materials Science and Engineering, University of New South Wales, SYDNEY, NSW \\ 2052, Australia. \\ ** Peregrine Semiconductor Australia Pty Ltd, HOMEBUSH, NSW 2140 Australia.
}

Silicon thin films with (100) orientation deposited on (1 1 i 02) sapphire thin films, termed SOS technology, have been used for many years in the production of integrated circuits. The density of defects in the silicon film following deposition is high, but significantly reduced by methods initially developed by Lau et al. [1]. This process has since been optimised to a level where very high quality, low defect density films are being produced suitable for high transistor density integrated circuit applications. The first step involves bombarding the silicon film with high energy silicon ions to make the film almost completely amorphous, except for a crystalline region in the silicon adjacent to the free surface. This can be seen in Figure 1A. Two annealing steps follow: The first at $\sim 550^{\circ} \mathrm{C}$ to recrystallise the amorphous silicon, from the residual crystalline material acting as a seed, by growth towards the silicon-sapphire interface. The second above $900^{\circ} \mathrm{C}$ to remove any remaining unstable defects.

Twins lying on $\{111\}$ have been found in the remaining crystalline silicon layer of the unannealed film following implantation as shown in Figure 1B. It has been largely accepted that these remaining twins do not grow back towards the silicon-sapphire interface because, during annealing at $550^{\circ} \mathrm{C}$, the twins are "pinched off" as the (100) silicon grows $\sim 10$ times faster than (221) material contained in the twins [2]. This paper argues that twins present in the crystalline region following implantation grow back significantly during heating to $\sim 550^{\circ} \mathrm{C}$ and are only pinched off when the material reaches this temperature.

TEM specimens were prepared using the 'liftout' technique using an FEI Nova 200 Nanolab focused ion beam miller. Cross sectional TEM samples were produced and analysed with a $200 \mathrm{kV}$ Philips CM-200 TEM.

Figures $1 \mathrm{C}$ and $1 \mathrm{D}$ show bright field TEM images corresponding to samples heated to $\sim 550^{\circ} \mathrm{C}$ within 0.5 and 20 mins respectively, before being air cooled. In Figure $1 \mathrm{C}$, twins are seen to extend fully through the crystalline region, which has expanded relative to the as-implanted material. Figure 1D shows that the twins continued to grow in parallel with the crystalline interface, but have extended further towards the sapphire than in the former case. Figure 1E shows a specimen heated over 20 mins and held at $\sim 550^{\circ} \mathrm{C}$ for 30 mins. The entire silcion film has recrystallised, but the twins have not propogated further during annealing at this temperature. The material adjacent to the sapphire, which is used for the manufacture of devices, is seen to be defect free.

This work shows that the ramp rate to $\sim 550^{\circ} \mathrm{C}$ used for re-crystallising silicon in Lau's process can be used to further isolate the $\{111\}$ twins to the outer surface of the SOS film. 
[1] S. S. Lau, et al., Appl. Phys. Lett. 34(1) (1979) 76.

[2] K. W. Carey, et al., J. Appl. Phys. 54(8) (1983) 4414.

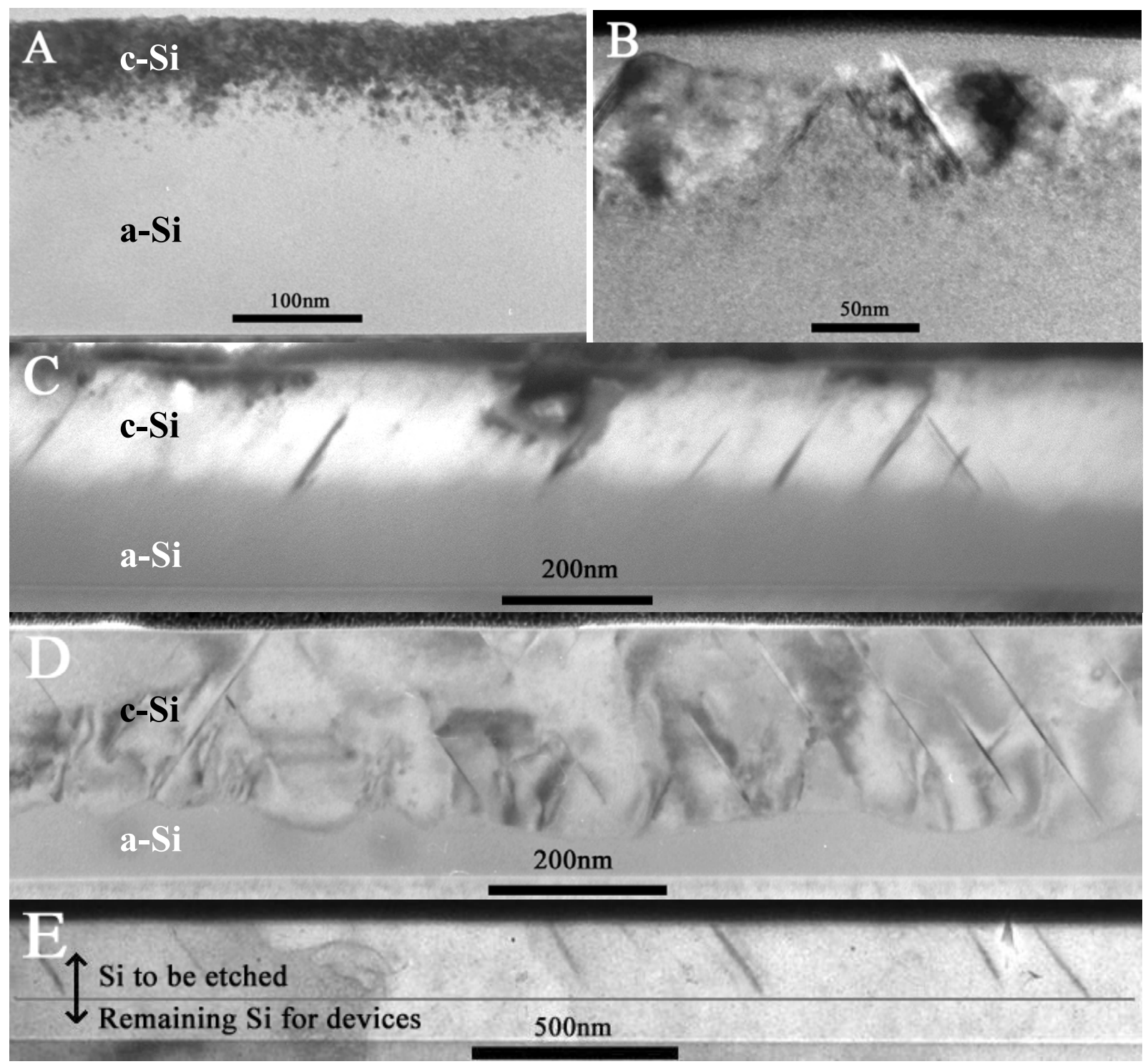

Figure 1: Bright field TEM images viewed parallel to the [110] zone axis: A) SOS film after implantation and before the regrowth anneal showing the remaining crystalline and amorphous regions. B) Higher magnification image of $1 \mathrm{~A}$ showing remaining twins. Heating and immediate cooling reaching $\sim 550^{\circ} \mathrm{C}$ after C)30 seconds and D) 20 minutes. E) Heating over 20 minutes, then held at $\sim 550^{\circ} \mathrm{C}$ for 30 minutes. The region that is subsequently etched to remove the remaining twins during conventional processing is indicated. 